\title{
A Modeling Method of Human Knee Joint Based on Biomechanics
}

\author{
Jin Gao ${ }^{1}$, Yajing Wang ${ }^{1}$, Yahui Cui, Xiaomin $\mathrm{Ji}^{1}$, Xupeng Wang ${ }^{1}$ \\ ${ }^{1}$ Department of Industrial Design, Xi'an University of Technology, Xi'an Shaanxi, 710048, China
}

\begin{abstract}
In order to establish an accurate model of human knee joint, which lays a foundation for the followup finite element analysis of knee joint and biomechanics analysis, the paper based on the theory of biomechanics and MRI, with the help of professional modeling software, provides a 3D geometric model modeling and materialization processing method of knee joint, and established a total knee joint model including femur, tibia, fibular, meniscus, cartilage, ligament and other related structures.
\end{abstract}

\section{Introduction}

Exercise is an important part of the daily activities of the human body, and the knee joint plays an important role in the movement of the human body. Due to the intensity of the movement, this area is often due to industrial production, accidents and sports injuries caused by injuries. According to the analysis of 2725 trauma cases in Beijing sports medicine research, $25.82 \%$ of them were knee joint injuries [1].

In recent years, biomechanical modelling and numerical simulation techniques have become an important means of biomechanical research on knee joints. With the development of scientific research and medical imaging technology in China, the accuracy of imaging diagnosis of knee osteoarthritis has been gradually improved. At present, X-ray and Magnetic Resonance Imaging (MRI) are commonly used in clinical diagnosis [2]. At present, a large number of researchers have studied the biomechanics of the knee joint through finite element analysis, and the research on the internal motion and stress law of the knee joint has become a hot topic in rehabilitation medicine and mechanical physiology. In 1980, Wismans et al. [3] successfully established the first quasi-statics model of knee joint based on human physiological and anatomical structure. In 1996, A.A.J. Goldsmith et al. [4] analysed the stress changes of knee cartilage by finite element method. The extraction of early knee joint model was difficult. Donahue et al. [5] initially collected bone structure information through CT scan, and established the meniscus finite element model after the meniscus information was collected by laser digital system. After establishing the knee joint model, Liu et al. [6] analysed the mechanical characteristics of the knee joint at $90^{\circ}$ flexion, and verified the accuracy of the model through the impact experiment. The load was $900 \mathrm{~N}$ on the quadriceps femoris, and the peak shear stress of the medial meniscus appeared in the middle and posterior part, about 3.34Mpa. However, this load value has not been verified.

In summary, biomechanics such as the internal motion and stress law of the knee joint can be studied by finite element analysis, which can simulate the mechanical changes of the knee joint under the condition of difficult samples. In this paper, it is proposed to conduct MRI scan of the knee joint of human body, and then reconstruct the knee joint model with the real two-dimensional tomography image, so as to provide a certain reference for the finite element numerical simulation of the knee joint model to obtain the stress changes of the meniscus cartilage and ligament in the knee joint.

\section{Knee joint structure and magnetic resonance imaging (MRI)}

MRI (Magnetic Resonance Imaging) is a type of tomography, without bone artifacts, can directly make cross-sectional, sagittal, coronal tomographic images [2]. The magnetic resonance phenomenon can be used to obtain electromagnetic signals from the human body, thereby reconstructing human body information. The technology has the advantages of good tissue resolution, non-invasive and non-radiation, and can present a more complete image of the knee joint tissue to help the doctor diagnose the knee joint disease. The gray-scale feature on the MRI image is that the stronger the magnetic resonance signal, the brighter the brightness. If the signal is large and the signal is weak, the brightness is also small, ranging from white to gray to black. No artifacts in CT detection. With the popularity of MRI, it has reached $40 \%$ of all relevant examinations. MRI is the most ideal way to collect geometric information of normal knee joints.

Common examination sequences of MRI include SE spin echo sequence, FSE/TSE fast spin echo sequence, IR fast inversion sequence, GE/GRE gradient echo sequence and EPI echo plane imaging. For the selection of the sequence, the sequence of knee MRI imaging selected fast 
spin echo sequence T2WI and PDWI, spin echo sequence T1WI. The contrast of PDWI is related to the density of hydrogen protons in the tissue. The meniscus contains fewer hydrogen-containing protons, and the low signal on PDWI can be clearly recognized. The principle of T2WI sequence imaging is long repetition and long echo time, and the ligament imaging is clearly displayed in T2WI sequence. The anterior and posterior cruciate ligaments are easily recognizable on the sagittal MRI, and the medial and lateral collateral ligaments are easily recognizable on the coronal plane.
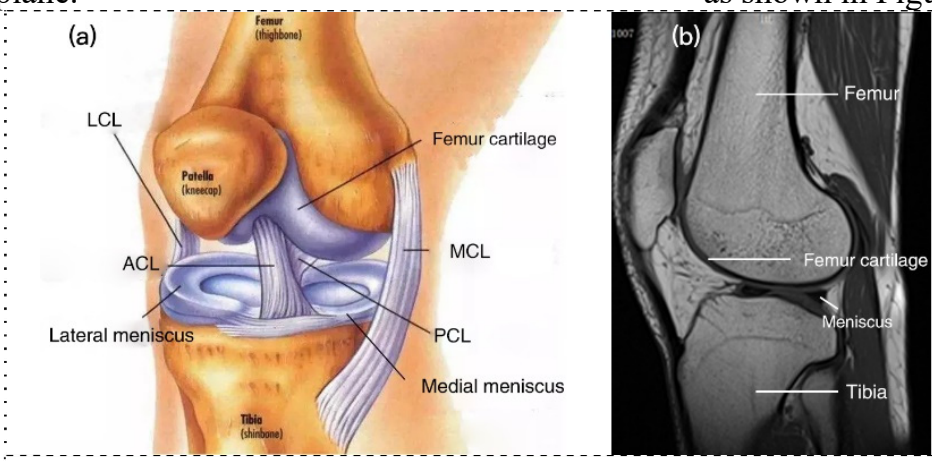

Figure 1. Comparison of knee joint physiological structure and MRI imaging.
In order to make the meniscus, ligament, femur, tibia and fibula easy to identify on DICOM, this study selected the coronal plane of T1WI sequence, the sagittal plane of PDWI sequence and the cross-sectional image of T2WI sequence for modelling. As shown in Fig. 1, the femur, tibia, articular cartilage, ligament and meniscus in the physiological structure of the knee joint have a one-to-one correspondence with the gray scale displayed on the MRI. The soft tissue magnetic resonance signal is weak, shown as black, and the bone signal is strong and bright. Larger, as shown in Figure 1.

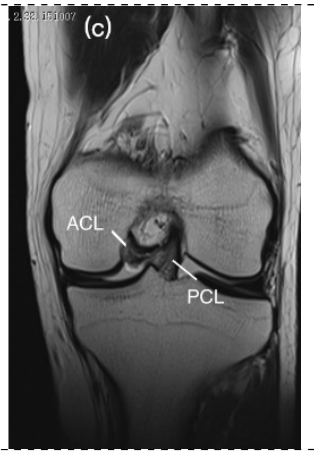

\section{Modeling environment and process of knee joint}

The modeling environment process of knee in this study are shown below, the knee modeling flowchart as shown in Figure 2.

(1) Nuclear magnetic resonance equipment: the scanning equipment is a $3.0 \mathrm{~T}$ superconducting MRI machine. The 3.0T has the characteristics of high field strength and high gradient field, which improves the signal-to-noise ratio and image spatial resolution. The experimental data collection was completed in the Second Affiliated Hospital of the Fourth Military Medical University.
(2) MIMICS 20.0 software: medical processing software, Belgium Leuven company, is a digital threedimensional interactive medical image control system, full name: Materialise 's interactive medical image control system;

(3) Geomagic Studio software: reverse engineering processing software, its high-precision surface can be converted by scanning the data of the three-dimensional model, and an accurate digital model can be automatically generated by scanning point cloud according to any physical parts;

(4) Rhinoceros software: It is a NURBS-based 3D modelling software launched by Robert McNeel in 1998. It is good at product appearance modelling, surface modelling and repair.

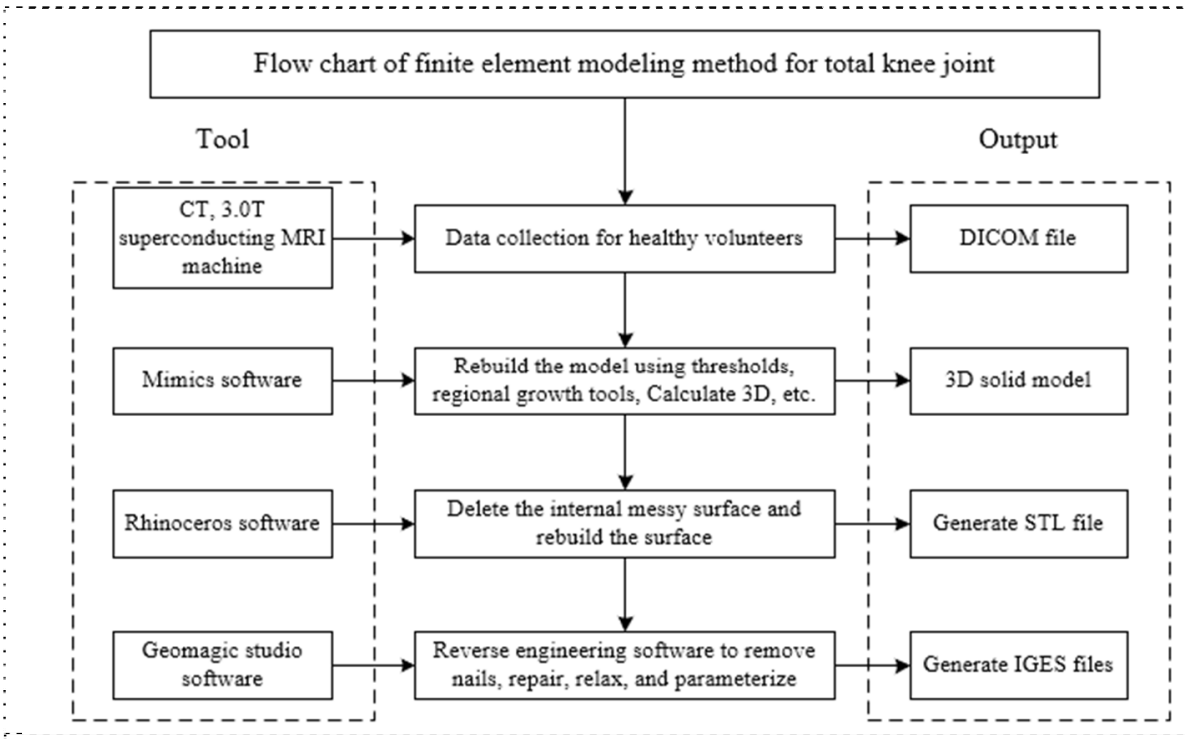

Figure 2. Knee Modelling Flowchart 


\section{Modeling method of knee}

In this study, the experimental volunteer was a $27-$ year-old healthy male weighing $75 \mathrm{~kg}$ and $180 \mathrm{~cm}$ tall. His knee joint was normal and he had no history of knee joint disease. The subject was in the supine position during the scan. To ensure the accuracy of the image, the knee joint of the left limb was fixed with a low-temperature thermoplastic plate during

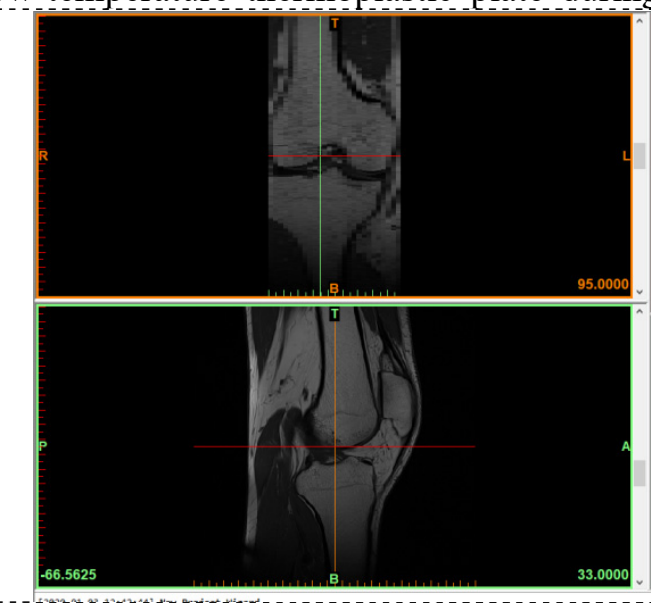

Figure 3. Two-dimensional image import Mimics software.

Open the DICOM file of the left limb named T1WI sequence. In this view, you can clearly see the cruciate ligament and the patellar ligament. Use the Profile Line tool in the MIMICS software to divide the required bone tissue in the sagittal, coronal and horizontal planes. According to the difference in gray value, use the threshold setting tool to extract the anterior cruciate ligament (ACL) and the posterior cross respectively Ligament (PCL) and patellar ligament. Use the regional growth tool (Region Growing) in the software for hot zone selection. If the structure to be reconstructed is similar to the gray value of the surrounding tissue, the $3 \mathrm{D}$ magnetic lasso tool can be used to outline the corresponding tissue, and then edited by the dynamic area growth tool to generate a three-dimensional model of the tissue; by the dynamic area growth tool After editing, the method of manual recognition is used for extraction. Each layer of the image must be edge segmented, redundant data removed, selective editing and hole filling processing, modified using Edit Masks function, and the Draw the scan. The thickness of the scan layer was $3 \mathrm{~mm}$ (slice thickness) refers to the single layer thickness).

\subsection{Modeling}

Import the scanned DICOM file into the medical processing software MIMICS 20.0, use the New Project Wizard command to open the DICOM file, and then define the direction to determine the coronal, crosssectional, and sagittal images, as shown in Figure 3.

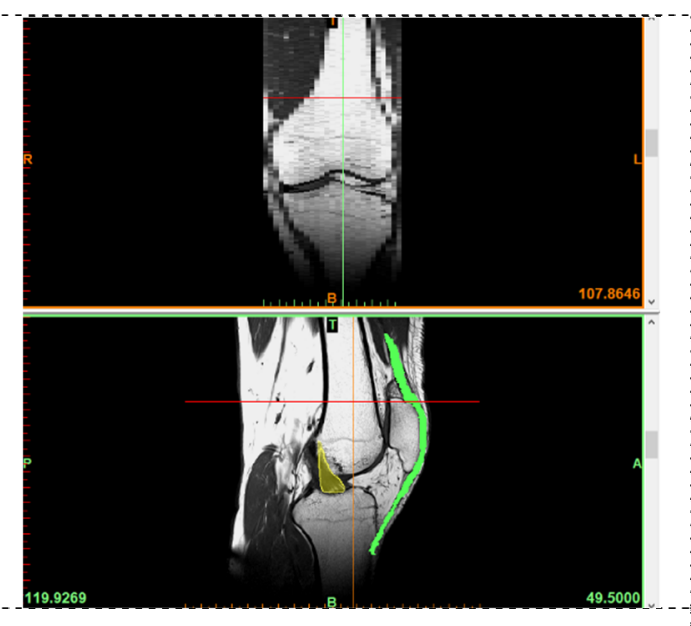

Figure 4. Ligament modelling process.

function to fill the vacancies in the tissue area. Partial and eraser function erase. Use the Calculate 3D command to directly convert 2D images into 3D models. The threedimensional model produced at this time is rough, with a lot of messy holes and extra triangular faces, which is far from the actual knee model. In the Mimics software, these poor quality areas need to be revised continuously until there are no extra noises in each view. Finally, a simple smoothing process is performed in Mimics to obtain the corresponding organization, and each organization is named after Export in STL format to obtain the ligament model shown in Figure 4.

Open the DICOM file of the left limb named PDWI sequence again. In this view, you can clearly see the femur, tibia, patella, fibula, meniscus, cartilage, lateral collateral ligament (LCL) and medial collateral ligament (MCL). Each organization adds a mask, and determines the later model based on the specific shape of the mask, as shown in Figure 5. 


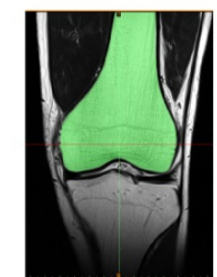

Femoral Mask

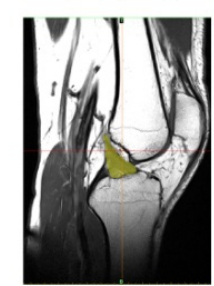

ACL Mask

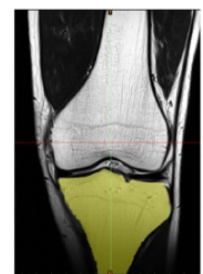

Tibia Mask

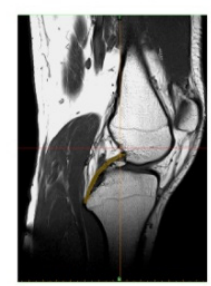

PCL Mask

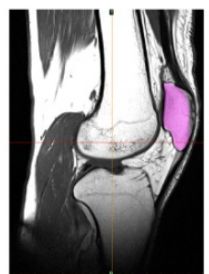

Patella Mask

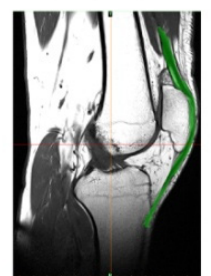

Patellar ligament Mask

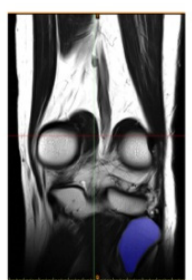

Fibula Mask

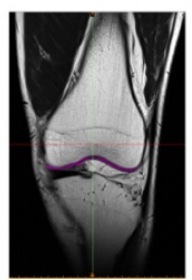

Femur cartilage Mask

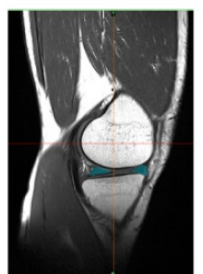

Meniscus Mask

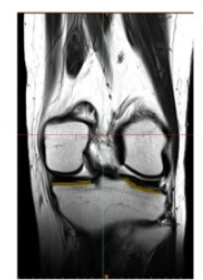

Tibia cartilage Mask

Figure 5. MIMICS modelling process

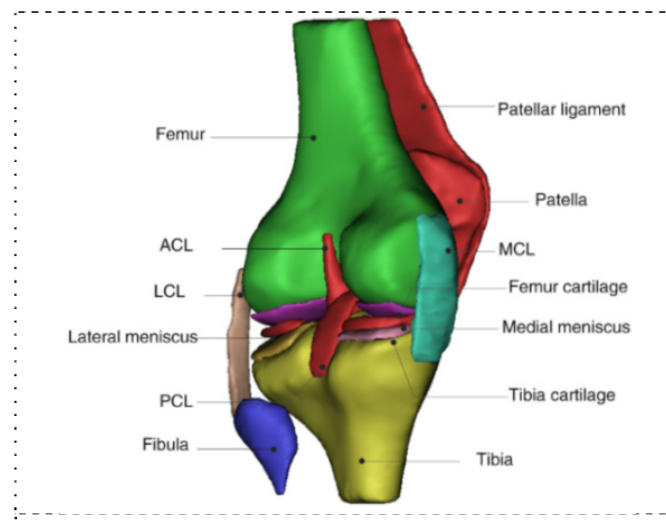

Figure 6. MIMICS full knee joint model.

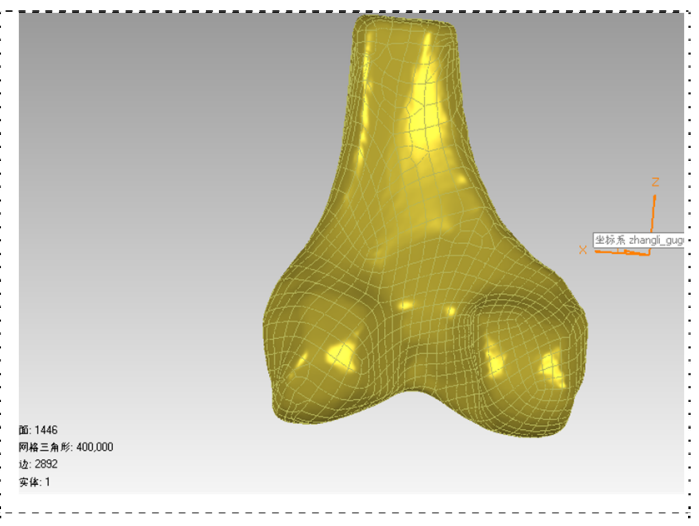

Figure 7. Femoral solidification process.
After adopting a series of modelling operations consistent with the above ligament modelling method, import the previously created ACL, PCL and patellar ligament into this MIMICS file, Select Point Registration to register in a point-to-point manner. Since the MRI file has its own coordinate system, the STL file will be automatically registered with the default original coordinate system when it is imported again. If there are untouched parts, you can manually select the registration. Finally, the full knee model shown in Fig. 6 is obtained.

\section{2 materialization}

After smoothing the model, MIMICS imports it into Geomagic Studio reverse engineering software for postprocessing. It is necessary to remove noise, simplify the mesh, repair the mesh, accurate the surface, construct the surface patch, construct the grid and fit the surface for each structure of the model Waiting for processing, because the model exported in MIMICS is a triangular patch format, it is easy to overlap each other where the model curvature is large. The first step is to simply process through the mesh doctor command, but the original shape of the model cannot be changed. During the molding process, the edge area is blurred, and the shape of the created 3D model is irregular. When using the grid doctor command, the smoothing will be directly ignored because of the small unit, which will seriously affect the accuracy of the model. Using manual repair will avoid this situation. For small triangles, you can use sandpaper to polish, delete and refill, smoothing. The second step is to parametrize the surface construction. You need to edit the contour line to construct the surface patch later. The third step is to click the precise surface command, choose to construct the surface patch, use the automatic estimation of the surface patch count, and check whether the paths intersect. If the surface intersects, it needs to be edited by the repair patch tool; then by constructing the grid, this is to make the model smoother and chamfer regular. Finally, the fitting tool is used to convert the surface to a NURBS surface, save it in IGES format, and reach the model Materialization, as shown in Fig. 7 of the femur model materialization process.

Surfaces generated in Geomagic Studio due to the continuity of the surface and some inherent defects in the original model generation process, caused the mesh to 
distort surface patches that are not in the same plane when generating the surface. These distorted surface patches will be used in later research Directly lead to analysis failure. In this paper, the model is imported into Rhinoceros for surface reconstruction. After the mesh is exploded and turned into a separate surface patch, the twisted surfaces are deleted one by one according to the research requirements, the control points of the surface are modified, and the surface operation is performed to achieve The surface is smooth and continuous until the inspection surface has no exposed edges and uneven surfaces. The final surface is a NURBS surface solid model, which is exported in IGES format.

\section{Conclusion}

In this paper, the DICOM file of the knee joint reconstruction was obtained by MRI scan of the left knee of the volunteer. Using MIMICS, Geomagic Studio and Rhinoceros software, according to a series of modeling steps, a three-dimensional geometric entity model of the human knee joint was constructed. The model includes the main structures of the knee joint such as femur, tibia, patella, fibula and its surface cartilage, medial meniscus, lateral meniscus, anterior cruciate ligament, posterior cruciate ligament, medial collateral ligament and lateral collateral ligament. Later, the model was processed a solid model of NURBS surface and provides a foundation for the follow-up study of the knee joint.

\section{Acknowledgments}

This work was supported by the Teaching research project from Xi'an University of Technology (Grant No. xjy1843) and Foundation from Xi'an University of Technology (Grant No. 106-451619016). The authors would like to express their appreciation to the agencies.

\section{References}

1. Huang Zubei, Zhu Hua, Peng Xiaochun. (2014) Research progress of imaging diagnosis of knee osteoarthritis [J]. Orthopaedics, 2014, 26 (6): 4345 .

2. Guo Jie. (1999) Biomechanical factors analysis of knee joint injury. Sports Science Research, 39 (1): 47-49.

3. Wismans J, Veldpaus F, Janssen J, et al. (1980) A Three-Dimensional Mathematical Model of the Knee-Joint. Journal of Biomechanics, 13(3): 667686.

4. A.A.J. Goldsmith, A. Hayes, S.E. Clift. (1996) Application of finite elements to the stress analysis of articular cartilage, Medical Engineering \& Physics, Volume 18, Issue 2, Pages 89-98, ISSN 1350-4533

5. Donahue TL, Hull ML, Rashid MM, et al. (2002) A finite element model of the human knee joint for the study of tibia femoral contact. J Biomech Eng, 124(3): 273-280.

6. Liu Junkang. (2017) Construction of human knee joint mechanical model and study of flexion motion mechanical characteristics. Science and Technology Bulletin, 33 (09): 246-250. 